\title{
Compatibilities between continuous semilattices
}

\begin{abstract}
Mykytsey O.Ya., Koporkh K.M.
We define compatibilities between continuous semilattices as Scott continuous functions from their pairwise cartesian products to $\{0,1\}$ that are zero preserving in each variable. It is shown that many specific kinds of mathematical objects can be regarded as compatibilities, among them monotonic predicates, Galois connections, completely distributive lattices, isotone mappings with images being chains, semilattice morphisms etc. Compatibility between compatibilities is also introduced, it is shown that conjugation of non-additive real-valued or lattice valued measures is its particular case. tibility.

Key words and phrases: conjugate capacity, continuous semilattice, monotonic predicate, compa-
\end{abstract}

Vasyl Stefanyk Precarpathian National University, 57 Shevchenka str., 76018, Ivano-Frankivsk, Ukraine

E-mail: omykytsei@gmail.com (Mykytsey O.Ya.), katerynka.k@gmail . com (Koporkh K.M.)

\section{Introduction}

The aim of this paper is to extend the class of compatibilities previously defined and investigated in [11]. It turned out that the original definition of compatibility was too restrictive, and many interesting rather close objects did not fit into it. We preserve the original class under the name "strong separating compatibilities", hence taking into consideration compatibilities that are not strong or not separating in the sense, which will be clarified further.

The motivation for such study was the discovery by the authors of hidden similarity between two rather distant fields of science. On the one hand, there is a theory of non-additive measures [5], with numerous applications to optimization, to game theory [2], to economy modeling [3], and decision making [7] etc. On the other hand, monotonic predicates are used extensively in denotational semantics of programming languages [8,9]. In both theories some kinds of duality appear, representing either conflict of interests, or distinction between things that are under our control and things that are controlled by someone else. Informally speaking, the key idea of this duality is that intentions of different parties are not always compatible.

We attempt to make this idea more formal through the notion of compatibility between elements of continuous semilattices. It is widely adopted [1] that continuous semilattices are appropriate tools to represent incomplete/impresize knowledge. There is a natural partial order on the set of all pieces of partial knowledge on a state of something: an information $x$ is greater or equal to an information $y$ if $x$ is more specific (restrictive) than $y$. Then the least element 0 means "anything is possible". Then a compatibility is a function of two arguments from semilattices $S$ and $S^{\prime}$ describing two observations, assumptions, or plans, that can take two possible values : $1=$ "incompatible" and $0=$ "probably compatible". 


\section{Preliminaries}

This paper is a continuation of [11], and it mostly follows its terminology and notation style. Although the reader can find in [11] a wide reference on continuous posets, we repeat small part of it here.

We adopt the following definitions and notation, which are consistent with [6]. All statements in this section are also numbered accordingly to the latter citation. From now on, semilattice means meet semilattice, if otherwise is not specified. If a poset contains a bottom (a top) element, then it is denoted by 0 (resp. by 1). A top element in a semilattice is also called a unit and denoted by 1 .

For a partial order $\leq$ on a set $X$, the relation $\widetilde{\leq}$, defined as $x \widetilde{\leq} y \Longleftrightarrow y \leq x$ for $x, y \in X$, is a partial order called opposite to $\leq$, and $(X, \leq)^{o p}$ denotes the poset $(X, \widetilde{\leq})$. If the original order $\leq$ is obvious, we write simply $X^{o p}$ for the reversed poset. We also apply ( ) to all notations to denote passing to the opposite order, i.e. write $\widetilde{X}=X^{o p}$, $\widetilde{\text { sup }}=$ inf, $\widetilde{0}=1$ etc.

For a subset $A$ of a poset $(X, \leq)$, we denote

$$
A \uparrow=\{x \in X \mid a \leq x \text { for some } a \in A\}, A \downarrow=\{x \in X \mid x \leq a \text { for some } a \in A\} .
$$

If $A=A \uparrow(A=A \downarrow)$, then a set $A$ is called upper (resp. lower).

A set $A$ in a poset $(X, \leq)$ is directed (filtered) if for all $x, y \in A$, there is $z \in A$ such that $x \leq z$, $y \leq z$ (resp. $z \leq x, z \leq y$ ). A poset is called directed complete (dcpo for short) if it has lowest upper bounds for all its directed subsets.

The Scott topology $\sigma(X)$ on a poset $X$ consists of all those $U \subseteq X$ that satisfy $x \in U \Leftrightarrow$ $U \cap D \neq \varnothing$ for every $\leq$-directed $D \subseteq X$ with a least upper bound $x$. Note that " $\Leftarrow$ " above implies $U=U \uparrow$.

A mapping $f$ between dcpo's $X$ and $Y$ is Scott continuous, i.e. continuous with respect to $\sigma(X)$ and $\sigma(Y)$, if and only if it preserves suprema of directed sets (cf. Proposition II.2-1).

Let $L$ be a poset. We say that $x$ is way below $y$ and write $x \ll y$ if and only if for all directed subsets $D \subseteq L$ such that $\sup D$ exists, the relation $y \leq \sup D$ implies the existence of $d \in D$ such that $x \leq d$. "Way-below" relation is transitive and antisymmetric.

A poset $L$ is called continuous if each element $y \in L$ is a least upper bound of a directed set of all $x \in L$ such that $x \ll y$. A domain is a continuous dcpo. If domain is a semilattice (a lattice), it is called a continuous semilattice (resp. a continuous lattice). Obviously a continuous lattice with a bottom element is a complete lattice.

The definition of completely distributive lattice can be found in [6]. For the reader to get necessary intuition, it is sufficient to say that completely distributive lattices are precisely distributive lattices that can be endowed with compact Hausdorff topology so that meet and join operations are continuous and there are local bases consisting of subsemilattices.

\section{Notion of compatibility}

Definition. Let $S, S^{\prime}$ be continuous semilattices with zero elements $0,0^{\prime}$ respectively. A mapping $P: S \times S^{\prime} \rightarrow\{0,1\}$ is called a compatibility between $S$ and $S^{\prime}$, if

(1) $P$ preserves zeros in both variables, i.e., $P(0, y)=P\left(x, 0^{\prime}\right)=0$ for all $x \in S, y \in S^{\prime}$;

(2) $P$ is Scott continuous. 
The set of all compatibities between $S$ and $S^{\prime}$ is denoted $C_{w}\left(S, S^{\prime}\right)$.

If, additionally,

(3) $P$ separates elements of $S$ and $S^{\prime}$, i.e.,

(3a) for each $x_{1}, x_{2} \in S$, if $P\left(x_{1}, y\right)=P\left(x_{2}, y\right)$ for all $y \in S^{\prime}$, then $x_{1}=x_{2}$;

(3b) for each $y_{1}, y_{2} \in S^{\prime}$, if $P\left(x, y_{1}\right)=P\left(x, y_{2}\right)$ for all $x \in S$, then $y_{1}=y_{2}$,

then $P$ is called a separating compatibility.

The above definition of (separating) compatibility is symmetric in the following sense: the mapping $P^{\prime}: S^{\prime} \times S \rightarrow\{0,1\}, P^{\prime}(y, x)=P(x, y)$ is a (separating) compatibility as well, and we call it the inverse compatibility. We also use notation $x P y \equiv P(x, y)$.

We can regard a compatibility $P: S \times S^{\prime} \rightarrow\{0,1\}$ as the characteristic mapping of a binary relation $P \subset S \times S^{\prime}$, hence it is natural to denote $x P=\left\{y \in S^{\prime} \mid x P y=1\right\}, P y=\{x \in S \mid$ $x P y=1\}$ for all $x \in S, y \in S^{\prime}$.

We interpret $P(x, y)=1$ as "pieces $x$ and $y$ of information are incompatible (cannot be valid simultaneously)". Thus probably the term "incompatibility" would be more appropriate. Compatibility $P: S \times S^{\prime} \rightarrow\{0,1\}$ is separating if and only if for all $x_{1} \neq x_{2}$ in $S$ there is $y \in S^{\prime}$ such that precisely one of $x_{i}$ is incompatible with $y$ with respect to $P$, similarly for $y_{1} \neq y_{2}$ in $S^{\prime}$ and $x \in S$. Then elements of $S^{\prime}$ can be considered "negative statements" on the state of a system observed in $S$ : having $y \in S^{\prime}$ and a separating compatibility $P$, we declare impossible all $x \in S$ such that $x P y=1$.

It is easy to observe that, of all compatibilies between $S$ and $S^{\prime}$, the greatest one is $R_{0}$ defined as follows:

$$
R_{0}(x, y)= \begin{cases}0, & \text { if } x=0 \text { or } y=0, \\ 1, & \text { if } x \neq 0 \text { and } y \neq 0 .\end{cases}
$$

Thus $C_{w}\left(S, S^{\prime}\right)=R_{0} \downarrow$ in the set $\left[S \times S^{\prime} \rightarrow\{0,1\}\right]$, i.e., in the set

$$
\left\{f: S \times S^{\prime} \rightarrow\{0,1\} \mid f \text { is Scott continuous }\right\},
$$

which is a completely distributive lattice due to [4, Theorem 4]. Then $C_{w}\left(S, S^{\prime}\right)$ is completely distributive as well, and $R_{0}$ is its greatest element. In the same set $\widetilde{C_{w}}\left(S, S^{\prime}\right)$ with the opposite order the element $R_{0}$ is the least one.

\section{Kinds and examples of compatibilities}

Recall that Scott continuity and zero preservation only are required in each argument for a compatibility. Imposing additional restrictions, we obtain narrower classes of compatibilities. We say that a compatibility $P: S \times S^{\prime}$ is meet preserving in the first argument if for all $x_{1}, x_{2} \in S, y \in S^{\prime}$ the equality $P\left(x_{1} \wedge x_{2}, y\right)=P\left(x_{1}, y\right) \wedge P\left(x_{2}, y\right)$ is valid. We denote $C_{\wedge}\left(S, S^{\prime}\right) \subset C_{w}\left(S, S^{\prime}\right)$ the subset of such compatibilities. Similarly $C_{\bullet \wedge}\left(S, S^{\prime}\right) \subset C_{w}\left(S, S^{\prime}\right)$ is the subset of compatibilities that are meet preserving in the second argument. The elements of the set $C_{\wedge \wedge}\left(S, S^{\prime}\right) \subset C_{w}\left(S, S^{\prime}\right)$, i.e., compatibilities that are meet preserving in both arguments, are called strong compatibilities. Meet preservation in the first argument means that, if $x_{1}$ and $x_{2}$ are incompatible (cannot be valid together) with $y$, then " $x_{1}$ or $x_{2}$ " is incompatible with $y$ as well, similarly for the second argument. This is probably the most important class of compatibilities that will be considered further in detail. 
Existence of all joins (pairwise suprema) in an arbitrary semilattice is not guaranteed. If a continuous semilattice $L$ with zero has all joins (i.e., $L$ is a continuous lattice, hence a complete lattice), then we call a compatibility $P: S \times L \rightarrow\{0,1\}$ join preserving in the second argument if $P\left(x, y_{1} \vee y_{2}\right)=P\left(x, y_{1}\right) \vee P\left(x, y_{2}\right)$ for all $x \in S, y_{1}, y_{2} \in L$. Denote $C_{\bullet \vee}(S, L)$ the set of such compatibilities. Then for all $x \in S$ the set $\{y \in L \mid P(x, y)=0\}$ contains zero, is Scott closed and directed. This implies that it has a greatest element $y_{0}$, and $\{y \in L \mid P(x, y)=0\}=y_{0} \downarrow$. The function $P$ is uniquely determined with the mapping $p: S \rightarrow L$ that takes each $x$ to the respective $y_{0}$, namely

$$
P(x, y)=\left\{\begin{array}{l}
0, y \leq p(x), \\
1, y \not \leq p(x),
\end{array} \quad x \in S, y \in L .\right.
$$

It is easy to show that $p$ is antitone, takes $0 \in S$ to $1 \in L$, and is Scott continuous as a mapping $S \rightarrow L^{o p}$, i.e., is an $L$-valued normalized monotonic predicate on $S$ [12]. Conversely, each $L$-valued normalized monotonic predicate $p: S \rightarrow L$ determines a compatibility $P \in C_{\bullet \vee}(S, L)$ with the above formula. Thus $C_{\bullet \vee}(S, L)$ can be identified with the set $M_{[L]} S$ of $L$-valued normalized monotonic predicates.

Let us clarify what is a narrower class $C_{\wedge \vee}(S, L)$ of compatibilities that are meet preserving in the first argument and join preserving in the second one. For each $P \in C_{\wedge \vee}(S, L)$ the respective function $p: S \rightarrow L$ should have the property: if $x_{1}, x_{2} \in S, y \in L, y \not \leq p\left(x_{1}\right)$ and $y \not \leq p\left(x_{2}\right)$, then $y \not \leq p\left(x_{1} \wedge x_{2}\right)$. For $p$ is antitone, $p\left(x_{1}\right) \leq p\left(x_{1} \wedge x_{2}\right), p\left(x_{2}\right) \leq p\left(x_{1} \wedge x_{2}\right)$, hence $y=p\left(x_{1}\right) \vee p\left(x_{2}\right) \leq p\left(x_{1} \wedge x_{2}\right)$. Therefore for this $y$ is either $y \leq p\left(x_{1}\right)$ or $y \leq p\left(x_{2}\right)$, i.e., either $p\left(x_{1}\right) \leq p\left(x_{2}\right)$ or $p\left(x_{2}\right) \leq p\left(x_{1}\right)$. This means that $P \in C_{\wedge \vee}(S, L)$ if and only if $p(S) \subset L$ is a chain (a linearly ordered subset). In particular, if $L$ is linearly ordered, e.g., $L=I$, then $C_{\wedge \vee}(S, L)=C_{\bullet \vee}(S, L)$.

Proceed further and consider two continuous lattices $L, L^{\prime}$ and a compatibility $P \in C_{\vee v}\left(L, L^{\prime}\right)$, i.e., we require join preservation in both arguments. By the above there is an antitone function $p: L \rightarrow L^{\prime}$ that maps $0 \in L$ to $1^{\prime} \in L^{\prime}$, suprema of directed sets to infima of their filtered images, and $P(x, y)=0 \Longleftrightarrow y \leq p(x)$. But similarly we can find an antitone map $q: L^{\prime} \rightarrow L$ that is Scott continuous as a mapping $L^{\prime} \rightarrow L^{o p}$, such that $0^{\prime} \in L^{\prime} \mapsto 1 \in L$, and $P(x, y)=0 \Longleftrightarrow x \leq q(x)$.

Definition 1 ([6]). If $S, S^{\prime}$ are posets and $p: S \rightarrow S^{\prime}, q: S^{\prime} \rightarrow S$ functions such that for all $s \in S$ and $s^{\prime} \in S^{\prime}$, the inequality $s \leq_{S} q\left(s^{\prime}\right)$ is valid if and only if $p(s) \leq_{S^{\prime}} s^{\prime}$, then $p$ is called a lower adjoint to $q$, resp. $q$ an upper adjoint to $p$, and the quadruple $\left(S, p, q, S^{\prime}\right)$ is called a Galois connection.

Such $p, q$ are isotone and each mapping of the adjoint pair $(p, q)$ is uniquely determined with the other one.

If for $p_{1}: S \rightarrow S^{\prime o p}$ and $p_{2}: S^{\prime o p} \rightarrow S$, the quadruple $\left(S, p_{1}, p_{2}, S^{\prime o p}\right)$ is a Galois connection, then $\left(S, p_{1}, p_{2}, S^{\prime}\right)$ is called a contravariant Galois connection.

Here is an equivalent definition.

Definition 2. If $S, S^{\prime}$ are posets and $p: S \rightarrow S^{\prime}, q: S^{\prime} \rightarrow S$ are functions such that for all $s \in S$ and $s^{\prime} \in S^{\prime}$, inequality $s \leq_{s} q\left(s^{\prime}\right)$ is valid if and only if $s^{\prime} \leq_{S^{\prime}} p(s)$, then $\left(S, p, q, S^{\prime}\right)$ is called a contravariant Galois connection. 
Such $p, q$ are antitone, and the latter definition is symmetric in that $\left(S^{\prime}, q, p, S\right)$ is a contravariant Galois connection as well.

For the constructed above functions $p: L \rightarrow L^{\prime}$ and $q: L^{\prime} \rightarrow L$ we have $y \leq p(x) \Leftrightarrow$ $x \leq q(y)$, hence $\left(L, p, q, L^{\prime}\right)$ is a contravariant Galois connection. And, conversely, each contravariant Galois connection $\left(L, p, q, L^{\prime}\right)$ between continuous lattices $L$ and $L^{\prime}$ determines a compatibility $P \in C_{\vee v}\left(L, L^{\prime}\right)$ in the above manner.

The simplest example of such compatibility arises from an arbitrary completely distributive lattice $L$ and the lattice $L^{\prime}=L^{o p}$ with the opposite order. Then $p=\mathbf{1}_{L}: L \rightarrow L^{o p}$ and $q=\mathbf{1}_{L}: L^{o p} \rightarrow L$ constitute a contravariant Galois connection that determines the standard compatibility

$$
P(x, y)=\left\{\begin{array}{l}
0, x \leq y, \\
1, x \not \leq y,
\end{array} \quad x \in L, y \in L^{o p} .\right.
$$

In particular, for $L=(I, \leq)$ we have $L^{o p}=(I, \geq) \cong(I, \leq)$, with the isomorphism taking each $x \in I$ to $1-x$. Then put $L^{\prime}=(I, \leq), P(x, y)=0 \Longleftrightarrow y \leq 1-x$, i.e.,

$$
P(x, y)=\left\{\begin{array}{l}
0, x+y \leq 1 \\
1, x+y>1
\end{array} \quad x, y \in I .\right.
$$

We can regard $P(x, y)$ as compatibility of claims $x$ and $y$ for a part of payoff: if the first player wants to obtain $x$ of the entire payoff and the second one wants to obtain $y$, and $x+y>1$, then the both players cannot be satisfied simultaneously.

The previous classes were defined through properties of cuts of a function $P$, i.e., through its dependencies on one argument when the other one is fixed. Now we determine a class $C_{\nearrow}(S, L) \subset C_{w}(S, L)$ of compatibilities with a condition that involves both arguments simultaneously: for all $x_{1}, x_{2} \in S, y \in L$

$$
P\left(x_{1} \wedge x_{2}, y\right)=\min \left\{P\left(x_{1}, y_{1}\right) \vee P\left(x_{2}, y_{2}\right) \mid y \leq y_{1} \vee y_{2}\right\}
$$

In other words, $P\left(x_{1} \wedge x_{2}, y\right)=0$ if and only if there are $y_{1}, y_{2} \in L$ such that $y \leq y_{1} \vee y_{2}$, $P\left(x_{1}, y_{1}\right)=P\left(x_{2}, y_{2}\right)=0$.

This condition implies

$$
P(x, y)=\min \left\{P\left(x, y_{1}\right) \vee P\left(x, y_{2}\right) \mid y \leq y_{1} \vee y_{2}\right\}
$$

i.e., $P\left(x, y_{1}\right)=P\left(x, y_{2}\right)=0 \Longrightarrow P\left(x, y_{1} \vee y_{2}\right)=0$, and $P$ is join preserving in the second argument. Hence, $P$ is determined by an antitone function $p: S \rightarrow L$ with the property $p\left(x_{1} \wedge x_{2}\right)=p\left(x_{1}\right) \vee p\left(x_{2}\right)$, i.e., it is a lower semilattice morphism as a mapping $p: S \rightarrow L^{o p}$, and, conversely, each Scott continuous lower semilattice morphism $p: S \rightarrow L^{o p}$ that preserves zero, in the above manner determines a compatibility from $C_{\nearrow}(S, L)$. Thus $C_{\nearrow}(S, L)$ can be identified with the subset $M_{\wedge[L]} S \subset M_{[L]} S$ of all $L$-valued normalized monotonic predicates on $S$ that take meets to joins.

Thus we observe that many specific kinds of mathematical objects can be regarded as compatibilities, among them monotonic predicates, Galois connections, completely distributive lattices (in fact double continuity is sufficient), isotone mappings with images being chains, semilattice morphisms etc. 


\section{Compatibilities between compatibilities}

For continuous semilattices $S_{1}, S_{2}, S_{1}^{\prime}$, $S_{2}^{\prime}$ with zeros fix compatibilities $P_{1} \in C_{w}\left(S_{1}, S_{1}^{\prime}\right), P_{2} \in$ $C_{w}\left(S_{2}, S_{2}^{\prime}\right)$. We define a mapping $P_{1} \otimes P_{2}: \tilde{C_{w}}\left(S_{1}, S_{2}\right) \times \tilde{C_{w}}\left(S_{1}^{\prime}, S_{2}^{\prime}\right) \rightarrow\{0,1\}$ as follows: for all $R \in C_{w}\left(S_{1}, S_{2}\right), R^{\prime} \in C_{w}\left(S_{1}^{\prime}, S_{2}^{\prime}\right)$ let

$P_{1} \otimes P_{2}\left(R, R^{\prime}\right)=\sup \left\{P_{1}\left(x, x^{\prime}\right) \cdot P_{2}\left(y, y^{\prime}\right) \mid x \in S_{1}, x^{\prime} \in S_{1}^{\prime}, y \in S_{2}, y^{\prime} \in S_{2}^{\prime}, x R y=0, x^{\prime} R^{\prime} y^{\prime}=0\right\}$.

Proposition 1. The mapping $P_{1} \otimes P_{2}$ is a compatibility between $\widetilde{C_{w}}\left(S_{1}, S_{2}\right)$ and $\widetilde{C_{w}}\left(S_{1}^{\prime}, S_{2}^{\prime}\right)$ that preserves joins in both arguments.

Proof. Denote $P=P_{1} \otimes P_{2}$. Then for the bottom element $R_{0}$ of the lattice $\widetilde{C_{w}}\left(S_{1}, S_{2}\right)$ and arbitrary $R^{\prime}$ we obtain

$$
P\left(R_{0}, R^{\prime}\right)=\sup \left\{P_{1}\left(x, x^{\prime}\right) \cdot P_{2}\left(y, y^{\prime}\right) \mid(x=0 \text { or } y=0) \text { and } P_{2}\left(x^{\prime}, y^{\prime}\right)=0\right\}=0,
$$

i.e., $P$ preserves zero in the first, and, analogously, in the second argument.

Let us verify Scott continuity of $P$ in the first argument. For an arbitrary directed set $\mathcal{D} \subset \widetilde{C_{w}}\left(S_{1}, S_{2}\right)$ denote $D_{0}=\widetilde{\sup } \mathcal{D}$. Then $D_{0}=\inf \mathcal{D}$ in $C_{w}\left(S_{1}, S_{2}\right.$.) For $x \in S_{1}$ and $y \in S_{2}$ we obtain $D_{0}(x, y)=\sup \left\{\inf \left\{D\left(x^{\prime}, y^{\prime}\right) \mid D \in \mathcal{D}\right\} \mid x^{\prime} \ll x, y^{\prime} \ll y\right\}$.

Compare $P\left(D_{0}, R^{\prime}\right)$ and $\sup \left\{P\left(D, R^{\prime}\right) \mid D \in \mathcal{D}\right\}$.

The assumption $\sup \left\{P\left(D, R^{\prime}\right), D \in \mathcal{D}\right\}=1$ implies existence $D_{1} \in \mathcal{D}, x_{0} \in S_{1}, x_{0}^{\prime} \in$ $S_{1}^{\prime}, y_{0} \in S_{2}, y_{0}^{\prime} \in S_{2}^{\prime}$ such that $D_{1}\left(x_{0}, y_{0}\right)=0, R^{\prime}\left(x_{0}^{\prime}, y_{0}^{\prime}\right)=0, P_{1}\left(x_{0}, x_{0}^{\prime}\right)=1, P_{2}\left(y_{0}, y_{0}^{\prime}\right)=1$. Then $\inf \left\{D\left(x_{0}, y_{0}\right) \mid D \in \mathcal{D}\right\}=0$, and, by isotonicity of all $D \in \mathcal{D}, \inf \{D(x, y) \mid D \in \mathcal{D}\}=0$ for all $x \ll x_{0}, y \ll y_{0}$. Hence, $\sup \left\{\inf \{D(x, y) \mid D \in \mathcal{D}\} \mid x \ll x_{0}, y \ll y_{0}\right\}=0$, i.e., $D_{0}\left(x_{0}, y_{0}\right)=0$. Therefore, $P\left(D_{0}, R^{\prime}\right)=1$.

Now, conversely, let $P\left(D_{0}, R^{\prime}\right)=1$, i.e., there are $x_{0} \in S_{1}, x_{0}^{\prime} \in S_{1}^{\prime}, y_{0} \in S_{2}, y_{0}^{\prime} \in S_{2}^{\prime}$ such that $D_{0}\left(x_{0}, y_{0}\right)=0, R^{\prime}\left(x_{0}^{\prime}, y_{0}^{\prime}\right)=0, P_{1}\left(x_{0}, x_{0}^{\prime}\right)=1, P_{2}\left(y_{0}, y_{0}^{\prime}\right)=1$. Scott continuity of $P_{1}$ and $P_{2}$ implies that there are $x \ll x_{0}, y \ll y_{0}, x^{\prime} \ll x_{0}^{\prime}, y^{\prime} \ll y_{0}^{\prime}$ such that $P_{1}\left(x, x^{\prime}\right)=P_{2}\left(y, y^{\prime}\right)=1$. Then, by the choice of $D_{0}$, there is $D_{1} \in \mathcal{D}$ such that $D_{1}(x, y)=0$. By monotonicity of $R^{\prime}$ we have also $R^{\prime}\left(x^{\prime}, y^{\prime}\right)=0$, hence $P\left(D_{1}, R^{\prime}\right)=1$, and $\sup \left\{P\left(D, R^{\prime}\right), D \in \mathcal{D}\right\}=1$.

Thus $P\left(D_{0}, R^{\prime}\right)$ and $\sup \left\{P\left(D, R^{\prime}\right) \mid D \in \mathcal{D}\right\}$ are either both equal to 1 or both equal to 0 , therefore are equal.

We have shown that $P$ is Scott continuous in each variable separately, hence is Scott continuous. This proves that $P=P_{1} \otimes P_{2}$ is a compatibility.

Let $R, Q \in C_{w}\left(S_{1}, S_{2}\right), R^{\prime} \in C_{w}\left(S_{1}^{\prime}, S_{2}^{\prime}\right)$. Recall that $R \widetilde{V} Q$ in $\widetilde{C}\left(S_{1}, S_{2}\right)$ is $R \wedge Q$ in $C\left(S_{1}, S_{2}\right)$, i.e., it is the pointwise minimum of the functions $P$ and $Q$. For $P=P_{1} \otimes P_{2}$ we have to prove the equality $P\left(R \wedge Q, R^{\prime}\right)=\max \left\{P\left(R, R^{\prime}\right), P\left(Q, R^{\prime}\right)\right\}$. For $P\left(R \wedge Q, R^{\prime}\right) \geq P\left(R, R^{\prime}\right)$, $P\left(R \wedge Q, R^{\prime}\right) \geq P\left(Q, R^{\prime}\right)$, we obtain $P\left(R \wedge Q, R^{\prime}\right) \geq \max \left\{P\left(R, R^{\prime}\right), P\left(Q, R^{\prime}\right)\right\}$. It is only to show that $P\left(R \wedge Q, R^{\prime}\right)=1, \max \left\{P\left(R, R^{\prime}\right), P\left(Q, R^{\prime}\right)\right\}=0$ is impossible.

Otherwise there are $x \in S_{1}, x^{\prime} \in S_{1}^{\prime}, y \in S_{2}, y^{\prime} \in S_{2}^{\prime}$ such that $R(x, y) \wedge R^{\prime}(x, y)=0$, $R^{\prime}\left(x^{\prime}, y^{\prime}\right)=0, P_{1}\left(x, x^{\prime}\right)=1, P_{2}\left(y, y^{\prime}\right)=1$. At least one of the equalities $R(x, y)=0$ or $R^{\prime}(x, y)=0$ must be valid, assume, e.g., that the first one is. By the assumption $P\left(R, R^{\prime}\right)=0$, hence for all $x \in S_{1}, x^{\prime} \in S_{1}^{\prime}, y \in S_{2}, y^{\prime} \in S_{2}^{\prime}$ the equalities $P_{1}\left(x, x^{\prime}\right)=1, R(x, y)=R^{\prime}\left(x^{\prime}, y^{\prime}\right)=0$ imply $P_{2}\left(y, y^{\prime}\right)=0$, which is a contradiction. Analogously $R^{\prime}(x, y)=1$ is impossible. Thus, $P$ is join preserving in the first, and, similarly, in the second argument.

By the above the compatibility $P=P_{1} \otimes P_{2}$ is then determined with a contravariant Galois connection that consists of antitone mappings $p: \widetilde{C_{w}}\left(S_{1}, S_{2}\right) \rightarrow \widetilde{C_{w}}\left(S_{1}^{\prime}, S_{2}^{\prime}\right), q: \widetilde{C_{w}}\left(S_{1}^{\prime}, S_{2}^{\prime}\right) \rightarrow$ 
$\widetilde{C_{w}}\left(S_{1}, S_{2}\right)$ such that $P\left(R, R^{\prime}\right)=0 \Longleftrightarrow R^{\prime} \widetilde{\leq} p(R) \Longleftrightarrow R \widetilde{\leq} q\left(R^{\prime}\right)$. Consider, e.g., the mapping $p$ :

$$
\begin{aligned}
p(R)= & \widetilde{\sup }\left\{R^{\prime} \in \tilde{C_{w}}\left(S_{1}^{\prime}, S_{2}^{\prime}\right) \mid\left(P_{1} \otimes P_{2}\right)\left(R, R^{\prime}\right)=0\right\} \\
= & \inf \left\{R^{\prime} \in C_{w}\left(S_{1}^{\prime}, S_{2}^{\prime}\right) \mid R^{\prime}\left(x^{\prime}, y^{\prime}\right)=1 \text { for all } x \in S_{1}, y \in S_{2},\right. \\
& \left.\quad x^{\prime} \in S_{1}^{\prime}, y^{\prime} \in S_{2}^{\prime} \text { such that } P_{1}\left(x, x^{\prime}\right)=1, P_{2}\left(y, y^{\prime}\right)=1, R(x, y)=0\right\} \\
= & \inf \left\{R^{\prime} \in C_{w}\left(S_{1}^{\prime}, S_{2}^{\prime}\right) \mid R^{\prime}\left(x^{\prime}, y^{\prime}\right) \geq \widehat{R}\left(x^{\prime}, y^{\prime}\right) \text { for all } x^{\prime} \in S_{1}^{\prime}, y^{\prime} \in S_{2}^{\prime}\right\},
\end{aligned}
$$

where $\widehat{R}\left(x^{\prime}, y^{\prime}\right)$ is defined as

$\widehat{R}\left(x^{\prime}, y^{\prime}\right)= \begin{cases}1, & \text { if there are } x \in S_{1}, y \in S_{2}, \text { such that } P_{1}\left(x, x^{\prime}\right)=1, P_{2}\left(y, y^{\prime}\right)=1, R(x, y)=0, \\ 0, & \text { if there are no such } x \in S_{1}, y \in S_{2} .\end{cases}$

Isotonicity and Scott continuity of $\widehat{R}$ in both arguments follow from isotonicity and Scott continuity of $P_{1}$ i $P_{2}$. It is straightforward to verify that $\widehat{R}$ is zero preserving in both arguments. Therefore $\widehat{R}$ is a compatibility, hence

$$
p(R)=\inf \left\{R^{\prime} \in C_{w}\left(S_{1}^{\prime}, S_{2}^{\prime}\right) \mid R^{\prime}\left(x^{\prime}, y^{\prime}\right) \geq \widehat{R}\left(x^{\prime}, y^{\prime}\right) \text { for all } x^{\prime} \in S_{1}^{\prime}, y^{\prime} \in S_{2}^{\prime}\right\}
$$

coincides with $\widehat{R}$. Analogously $q\left(R^{\prime}\right)=\widehat{R}^{\prime}$, where

$\widehat{R^{\prime}}(x, y)=\left\{\begin{array}{l}1, \text { if there are } x^{\prime} \in S_{1}^{\prime}, y^{\prime} \in S_{2}^{\prime}, \text { such that } P_{1}\left(x, x^{\prime}\right)=1, P_{2}\left(y, y^{\prime}\right)=1, R^{\prime}\left(x^{\prime}, y^{\prime}\right)=0, \\ 0, \text { if there are no such } x^{\prime} \in S_{1}^{\prime}, y^{\prime} \in S_{2}^{\prime} .\end{array}\right.$

We call $\widehat{R}$ and $\widehat{R}^{\prime}$ the conjugate compatibilities to the compatibilities $R$ and $R^{\prime}$ respectively. Then $R \widetilde{\leq} q(p(R))$, i.e., $R \geq q(p(R))$. The compatibilities of the form $R=q\left(R^{\prime}\right)$ are precisely those satisfying the equality $q \circ p(R)=R$.

\section{Strong compatibilities}

Exercise IV.2-21 [6] implies that, for a continuous semilattice $S$ with a zero, the set $S^{\wedge}$ of all Scott open filters in $S$ except $S$ itself, but including $\varnothing$, when ordered by inclusion, is a continuous semilattice with the bottom element $\varnothing$. Moreover, the continuous semilattice $\left(S^{\wedge}\right)^{\wedge}$ with a zero is isomorphic to $S$, with an isomorphism $i: S \rightarrow\left(S^{\wedge}\right)^{\wedge}$ being $i(x)=\left\{\mathrm{F} \in S^{\wedge} \mid x \in \mathrm{F}\right\}$.

It was proved by the first author in [11] that the mapping $P: S \times S^{\wedge} \rightarrow\{0,1\}$,

$$
P(x, \mathrm{~F})=\left\{\begin{array}{l}
1, x \in \mathrm{F}, \\
0, x \notin \mathrm{F},
\end{array} \quad x \in S, \mathrm{~F} \in S^{\wedge},\right.
$$

is a strong separating compatibility. In fact all strong separating compatibilities are of this form, namely the following assertion is true.

Proposition 2 ([11]). Let $S, S^{\prime}$ be continuous semilattices with zeros. If a mapping $P: S \times S^{\prime} \rightarrow\{0,1\}$ is a strong separating compatibility, then the mapping $i$ that takes each $x \in S$ to $x P=\{y \in S \mid P(x, y)=1\}$ is an isomorphism $S \rightarrow S^{\prime \wedge}$. And, conversely, each isomorphism $i: S \rightarrow S^{\prime \wedge}$ is determined by a unique strong separating compatibility $P: S \times S^{\prime} \rightarrow\{0,1\}$ by the above formula.

Let us consider a strong separating compatibility that is well known (although not under the name proposed in this paper) and widely used in the theory of non-additive measures. 
Example 1. Recall that the set $\exp X$ of all non-empty closed subsets of a compactum $X$, when ordered by reverse inclusion, form a complete continuous semilattice $\exp _{\supset} X$, with a bottom element $X$ (cf. Exercise VI.3-18 [6]). For all $A, B \in \exp _{\supset} X$ denote

$$
P(A, B)= \begin{cases}0, & A \cap B \neq \varnothing, \\ 1, & A \cap B=\varnothing .\end{cases}
$$

We leave to the reader a verification that $P$ is a strong separating compatibility between $\exp _{\supset} X$ and $\exp _{\supset} X$.

\section{Conjugate capacities and conjugate compatibilities}

Monotonic $L$-valued predicates on a continuous semilattice $S$, i.e., antitone mappings from $S$ to a completely distributive lattice $L$ that take $0 \in S$ to $1 \in L$ and directed suprema in $S$ to filtered infima in $L$, are also known [10] as $L$-valued capacities on the semilattice $S$. To understand why is this name used, consider the case $S=\exp _{\supset} X$ and $L=I$. Then an $I$-valued monotonic predicate $c$ on $\exp _{\supset} X$ takes each non-empty subset $A$ of a compactum $X$ to a number $c(A) \in[0,1]$, so that

(1) $c(X)=1$;

(2) $A \subset B$ implies $c(A) \leq c(B)$;

(3) for a filtered family $\left(A_{i}\right)_{i \in \mathcal{I}}$ of non-empty closed subsets $A_{i} \subset X$

$$
c\left(\bigcap_{i \in \mathcal{I}} A_{i}\right)=\inf _{i \in \mathcal{I}} c\left(A_{i}\right)
$$

If we additionally define

(0) $c(\varnothing)=0$

and observe that (3), which is called $\tau$-smoothness, is equivalent to upper semicontinuity

(3a) for each $A \underset{\mathrm{cl}}{\subset} X$ and $\varepsilon>0$ there is $U \underset{\text { op }}{\subset} X, U \supset A$ such that $c(B)<c(A)+\varepsilon$ for all $B \underset{\mathrm{cl}}{\subset} X, B \subset U$,

then (0), (1), (2), and (3a) are precisely the definition of regular normalized capacity [13] (or normalized non-additive measure) on a compactum X. It lacks additivity

(4) $c(A \cup B)+c(A \cap B)=c(A)+c(B)$ for all $A, B \underset{\mathrm{cl}}{\subset} X$,

to be a probability measure on a compactum $X$.

Hence lattice-valued capacities on continuous semilattices are natural generalizations of real-valued capacities on compacta.

For a normalized $I$-valued capacity $c$ on a compactum $X$, the function $\widetilde{c}: \exp X \cup\{\varnothing\} \rightarrow I$,

$$
\widetilde{c}(A)=1-\sup \{c(B) \mid B \underset{\mathrm{cl}}{\subset} X, A \cap B=\varnothing\},
$$

is a normalized $I$-valued capacity on $X$ as well, and is called the conjugate capacity to $c$. If $c$ is additive, then $\widetilde{c}=c$ (probability measures are self-conjugate), but generally it is not the case. 
Analogously the conjugate capacity can be defined for any $L$-valued capacity $c$ on a continuous semilattice $S$ with zero, such that a strong separating compatibility $P: S \times S^{\prime} \rightarrow\{0,1\}$ is fixed. Namely, determine a function $\widetilde{c}: S^{\prime} \rightarrow \widetilde{L}$ with the formula

$$
\widetilde{c}\left(x^{\prime}\right)=\sup \left\{c(x) \mid P\left(x, x^{\prime}\right)=1, x \in S\right\}, \quad x^{\prime} \in S^{\prime} .
$$

Then [11] $\widetilde{c}$ is a normalized $\widetilde{L}$-valued capacity on $S^{\prime}$. Moreover, if the inverse compatibility $P^{\prime}: S^{\prime} \times S \rightarrow\{0,1\}$ is used to determine the "double conjugate" $\widetilde{\widetilde{c}}$, then

$$
\widetilde{\widetilde{c}}(x)= \begin{cases}c(x), & x \neq 0, \\ 1, & x=0,\end{cases}
$$

for all $x \in S$.

It implies that $\widetilde{\widetilde{c}}=c$ if and only if $c$ is normalized.

Recall that we identify $c$ with the compatibility between $S$ and $L$ defined as

$$
R(x, \alpha)= \begin{cases}0, & \text { if } \alpha \leq c(x), \\ 1, & \text { if } \alpha \leq \leq c(x) .\end{cases}
$$

Analogously a normalized $\widetilde{L}$-valued capacity on $S^{\prime}$ is identified with the following compatibility $R^{\prime}$ between $S^{\prime}$ and $\tilde{L}$

$$
R^{\prime}\left(x^{\prime}, \beta\right)= \begin{cases}0, & \text { if } \beta \widetilde{\leq} c^{\prime}(y), \\ 1, & \text { if } \beta \widetilde{z} c^{\prime}(y) .\end{cases}
$$

Using the standard compatibility between $L$ and $\widetilde{L}$

$$
P_{L}(x, y)=\left\{\begin{array}{l}
0, x \leq y, \\
1, x \not \leq y,
\end{array} \quad x \in L, y \in \widetilde{L},\right.
$$

we obtain the compatibility $P \otimes P_{L}$ between $M_{[L]} S \cong C_{\bullet \vee}(S, L)$ and $M_{[\tilde{L}]} S^{\prime} \cong C_{\bullet \vee}\left(S^{\prime}, \tilde{L}\right)$, and $P \otimes P_{L}\left(R, R^{\prime}\right)=0$ if and only if $c(x) \leq c^{\prime}\left(x^{\prime}\right)$ for all $x \in S, x^{\prime} \in S^{\prime}$ such that $P\left(x, x^{\prime}\right)=1$. Then we call $c$ and $c^{\prime}$ compatible. The conjugate capacity $\widetilde{c}$ is the greatest normalized $\widetilde{L}$-valued capacity that is compatible with $c$, and it corresponds to $\widehat{R}$, which is the greatest compatible with $R$ compatibility that preserves joins in the second argument.

Proposition 3. If a normalized L-valued capacity $c$ on a continuous semilattice $S$ with zero determines the compatibility $R$ between $S$ and $L, P$ is a strong separating compatibility between $S$ and $S^{\prime}, P_{L}$ is the standard compatibility between $L$ and $\widetilde{L}$, then the conjugate capacity $\widetilde{c}: S^{\prime} \rightarrow \widetilde{L}$ determines the compatibility $\widehat{R}$ between $S^{\prime}$ and $\widetilde{L}$ that is conjugate to $R$ with respect to $P \otimes P_{L}$.

Conjugation of capacities (of monotonic predicates) is thus a restriction of conjugation of compatibilities.

\section{Conclusions and future work}

We hope that the reader is convinced now that compatibilities are quite natural things and worth investigating. In fact, much more questions arise that are answered in this paper. E.g., we know that "double conjugate" of a normalized capacity is the same capacity, i.e., both 
components of the respective contravariant Galois connection are antiisomorphisms. It is not the case for arbitrary compatibilities, hence it would be interesting to establish conditions for these components being injective/surjective, and to describe their images in general case.

Some relations of compatibilities to idempotent linear algebra [12] have been uncovered, but much has also to be done in this direction. We leave this for future publications.

\section{References}

[1] Edalat A. Domains for computation in mathematics, physics and exact real arithmetic. Bull. Symb. Logic 1997, 3 (4), 401-452.

[2] Eichberger J., Kelsey D. Non-additive beliefs and strategic equilibria. Games Econom. Behav. 2000, 30, $183-215$. doi:10.1006/game.1998.0724

[3] Epstein L., Wang T. "Beliefs about beliefs" without probabilities. Econometrica 1996, 64 (5), $1343-1373$. doi: $10.2307 / 2171834$

[4] Erné M. Z-distributive function spaces. Hannover, Leibnitz University, 1998 (Preprint).

[5] Friedman N., Halpern J. Plausibility Measures: A User's Guide. In: Proceedings of the 11th Annual Conference on Uncertainty in Artificial Intelligence (UAI-95), Morgan Kaufmann, San Francisco, 1995, 175-184.

[6] Gierz G., Hofmann K.H., Keimel K., Lawson J.D., Mislove M., Scott D.S. Continuous Lattices and Domains. Encyclopedia of Mathematics and its Applications, vol. 93, Cambridge University Press, 2003.

[7] Grabisch M. Fuzzy integral in multicriteria decision making. Fuzzy Sets and Systems 1995, 69, $279-298$.

[8] Heckmann R. Quantitative semantics, topology, and possibility measures. Topol. Appl. 1998, 89, 151-178.

[9] Ying M. Reasoning about probabilistic sequential programs in a probabilistic logic. Acta Inf. 2003, 39 (5), $315-389$. doi:10.1007/s00236-003-0113-z

[10] Nykyforchyn O.R. Capacities with values in compact Hausdorff lattices. Appl. Categ. Structures 2008,15 (3), 243-257. doi:10.1007/s10485-007-9061-z

[11] Nykyforchyn O. Conjugate measures on semilattices. Visnyk of the Lviv Univ. Ser. Mech. Math. 2010, 72, 221231.

[12] Nykyforchyn O. Ambiguous representations of semilattices, imperfect information, and predicate transformers. Order 2020, 37, 319-339. doi:10.1007/s11083-019-09508-0

[13] Nykyforchyn O.R., Repovš D. Inclusion hyperspaces and capacities on Tychonoff spaces: functors and monads. Topology Appl. 2010, 157 (15), 2421-2434. doi:10.1016/j.topol.2010.07.032

Received 20.11.2020

Микицей О.Я., Копорх К.М. Сумісності між неперервними напівгратками // Карпатські матем. публ. - 2021. - Т.13, №1. - С. 5-14.

Ми означуємо сумісності між неперервними напівгратками як неперервні за Скоттом функції з їх попарних добутків у $\{0,1\}$, які зберігають нуль по кожній змінній. Показано, що багато видів математичних об'єктів можна розглядати як сумісності, зокрема, монотонні предикати, зв' язки Галуа, цілком дистрибутивні гратки, ізотонні відображення, образи якихє ланцюгами, морфізми напівграток та ін. Запроваджено також сумісність між сумісностями і показано, що спряження дійснозначних чи граткозначних неадитивних мір є їі частинним випадком.

Ключові слова і фрази: спряжена ємність, неперервна напівгратка, монотонний предикат, сумісність. 